\title{
Scalar-pseudoscalar meson spectrum in SU(3) PNJL model
}

\section{P. Costa*}

Departamento de Física, Universidade de Coimbra, Portugal

E.S.T.G., Instituto Politécnico de Leiria, Morro do Lena-Alto do Vieiro, 2411-901 Leiria,

Portugal

E-mail: pcosta@teor.fis.uc.pt

\section{C. Ruivo}

Departamento de Física, Universidade de Coimbra, Portugal

E-mail: mariaeteor.fis.uc.pt

\section{A. de Sousa}

Departamento de Física, Universidade de Coimbra, Portugal

E-mail: celia@teor.fis.uc.pt

\section{H. Hansen}

Univ.Lyon/UCBL, CNRS/IN2P3, IPNL, 69622 Villeurbanne Cedex, France

E-mail: hansen@ipnl.in2p3.fr

\section{W.M. Alberico}

Dipartimento di Fisica Teorica, University of Torino and INFN, Sezione di Torino, via P. Giuria 1, I-10125 Torino, Italy

E-mail: alberico@to.infn.it

\begin{abstract}
We investigate the modifications of mesonic properties in a hot medium having in mind to use the mesonic modes as a tool to analyze the restoration of chiral and axial symmetries in the context of the SU(3) Polyakov-Nambu-Jona-Lasinio (PNJL) model. The results lead to the conclusion that the effects of the Polyakov loop are fundamental for reproducing lattice findings. It is found that the restoration of chiral symmetry in the PNJL model occurs in a small range of temperature (smaller that in the Nambu-Jona-Lasinio (NJL)), and the topological susceptibility reproduces well the lattice results around $T / T_{c}^{\chi} \approx 1.0$.
\end{abstract}

8th Conference Quark Confinement and the Hadron Spectrum September 1-6, 2008

Mainz. Germany

\footnotetext{
${ }^{*}$ Speaker.
} 
The study of the modifications of particles propagating in a hot or dense matter is an interesting tool to investigate the restoration of chiral and axial symmetries. Understanding the restoration of symmetries and deconfinement, which are expected to occur under extreme conditions (high density and/or temperature), and, more recently, the possible survival of bound states in the deconfined phase of QCD, is a challenging problem [1,2].

In Ref. [3] the study of meson properties, in the SU(2) sector, around the critical region, was performed in the framework of the modified Nambu-Jona-Lasinio model including the Polyakov loop (the so-called PNJL model) $[4,5,6]$. The inclusion of the Polyakov loop is fundamental for reproducing lattice data concerning QCD thermodynamics [6], once it originates a suppression of the unwanted quark contributions to the thermodynamics below the critical temperature.

In this paper, we generalize the investigation to the SU(3) sector. Since the scalar mesons $\left(\sigma, f_{0}, a_{0}\right.$ and $\left.K_{0}^{*}\right)$ can be considered as chiral partners of the pseudoscalar nonet $\left(\eta, \eta^{\prime}, \pi^{0}\right.$ and $K$ ), it is interesting to see how the Polyakov loop affects the behavior of these mesons, having in mind the investigation of effective restoration of chiral symmetry.

The flavor mixing induced by the presence of the axial anomaly causes a violation of the OZI rule, both for scalar and pseudoscalar mesons, hence the restoration of axial symmetry should have relevant consequences on the phenomenology of the mesonic mixing angles, as well as on the topological susceptibility.

We perform our calculations in the framework of an extended SU(3) PNJL Lagrangian, which contains the 't Hooft instanton induced interaction term that breaks the $U_{A}(1)$ symmetry [7]:

$$
\begin{aligned}
\mathscr{L}_{P N J L} & =\bar{q}\left(i \gamma^{\mu} D_{\mu}-\hat{m}\right) q+\frac{1}{2} g_{S} \sum_{a=0}^{8}\left[\left(\bar{q} \lambda^{a} q\right)^{2}+\left(\bar{q} i \gamma_{5} \lambda^{a} q\right)^{2}\right] \\
& +g_{D}\left\{\operatorname{det}\left[\bar{q}\left(1+\gamma_{5}\right) q\right]+\operatorname{det}\left[\bar{q}\left(1-\gamma_{5}\right) q\right]\right\}-\mathscr{U}(\Phi, \bar{\Phi} ; T) .
\end{aligned}
$$

The Polyakov loop is an order parameter for the restoration of the $\mathbb{Z}_{3}$ (the center of $\mathrm{SU}_{c}(3)$ ) symmetry of QCD and is related to the deconfinement phase transition: $\mathbb{Z}_{3}$ is broken in the deconfined phase $(\Phi \rightarrow 1)$ and restored in the confined one $(\Phi \rightarrow 0)$ [8].

We start our study by analyzing the masses of the strange and non-strange quarks as functions of the temperature (on Fig. 1 the dressed quark masses $2 M_{u}, 2 M_{S}$ and $M_{u}+M_{S}$ are displayed). We verify that the mass of the light quarks drops to the current quark mass, indicating a smooth crossover from the chiral broken to an approximate chiral symmetric phase. This dropping is more pronounced in the PNJL model than in the NJL one. The strange quark mass presents a very similar behavior, with a significant decrease above the chiral transition temperature $T_{c}^{\chi}$, however its mass is still far away from the strange current quark mass. As usual chiral symmetry shows a slow tendency to get restored in the strange sector, however this tendency is faster in the PNJL model. As in the NJL model [9], once $m_{u}=m_{d}<m_{s}$, the (sub)group SU(2) $\otimes \mathrm{SU}(2)$ is a much better symmetry of the Lagrangian (1).

Nevertheless, the fact that the masses of the quarks drop faster around $T_{c}^{\chi}$ in PNJL model is important for the mesonic properties (for example it could modify the survival of mesonic bound states in the plasma phase). Besides, due to the strangeness content of some mesons, the behavior of the strange quark (modified by the Polyakov loop) is important for their properties, as well as for other observables related to the axial anomaly (as noticed in [12], topological susceptibility is strongly influenced by the strange sector). 


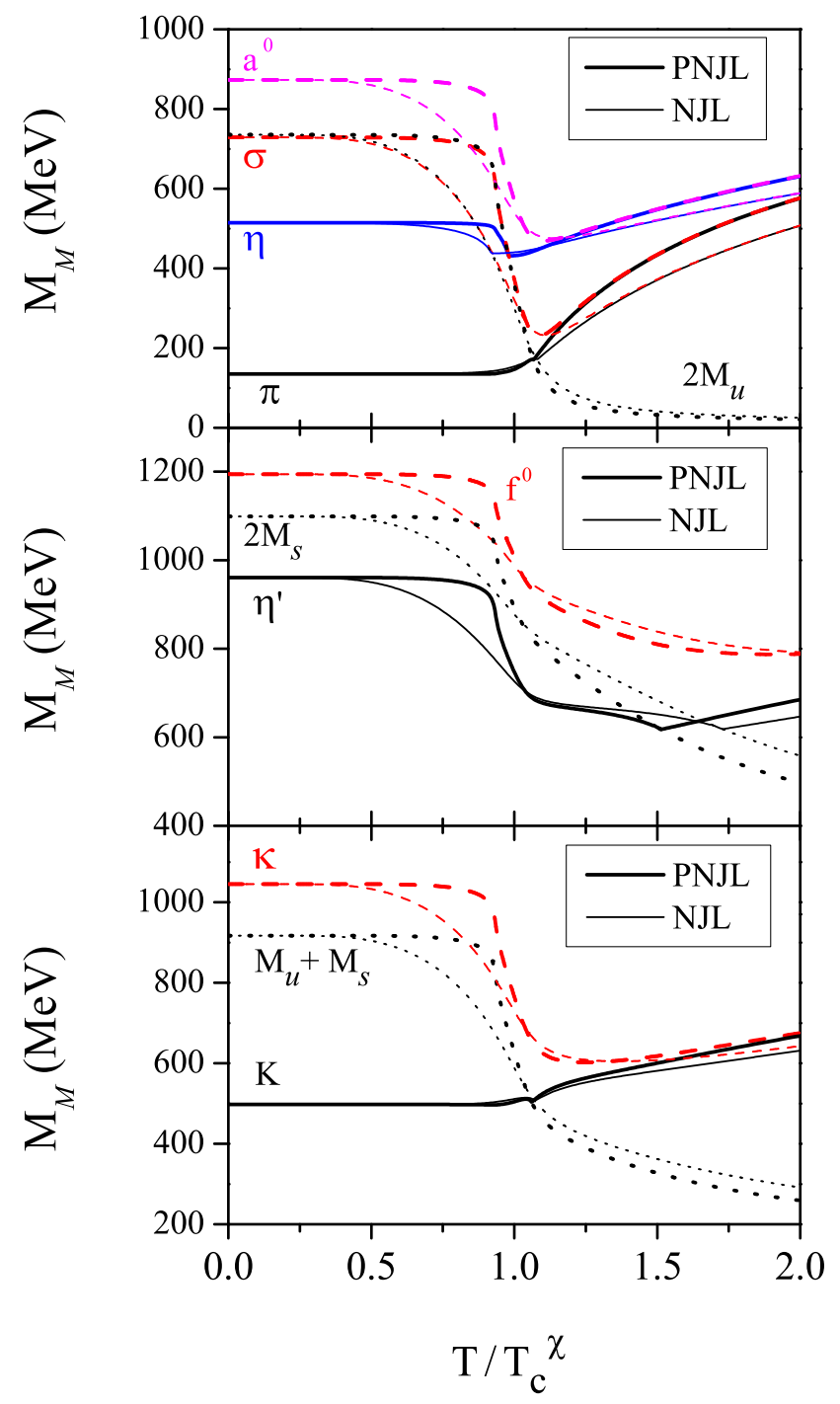

Figure 1: Comparison of the pseudoscalar and scalar meson masses in PNJL (thick lines) and NJL (thin lines) models as functions of the reduced temperature $T / T_{c}^{\chi}$. In the upper panel the $a_{0}$ (dashed line), $\sigma$ (dot-dashed line), $\eta$ and $\pi$ (continuous lines) are shown, together with $2 M_{u}$ (dotted lines). In the middle panel $f_{0}$ (dashed line) and $\eta^{\prime}$ (continuous line) are shown and compared with $2 M_{s}$ (dotted line). In the lower panel the $\kappa$ (dashed line) and $K$ (continuous line) masses are compared with $M_{u}+M_{s}$ (dotted line).

Let us now analyze the behavior of the mesons in connection with possible restoration of symmetries. It can be seen in Fig. 1 (upper panel) that the partners $(\pi, \sigma)$ and $\left(\eta, a_{0}\right)$ become degenerate at almost the same temperature. In both models, this behavior indicates the effective restoration of chiral symmetry in the non-strange sector.

On the contrary, the $\eta^{\prime}$ and $f_{0}$ masses (Fig. 1 middle panel) do not show a tendency to converge in the region of temperatures studied, a behavior that reflects the fact that chiral symmetry does not get restored in the strange sector. Finally, we focus on the $\kappa$-meson (lower panel): it is always an unbound, resonant state and, as the temperature increases, it tends to become degenerate in mass 
with the $K$-meson, but at larger temperatures (of the order of $1.5 T_{c}^{\chi}$ in PNJL, and higher in NJL). In summary, the masses of the $\sigma$ and $\eta$ (that become less strange as the temperature increases and the mixing in the strange sector decreases) converge, respectively, with those of the non-strange, $\pi^{0}$ and $a_{0}$. The convergence of the chiral partners $\kappa$ and $K$ (Fig. 1 lower panel), that have a $\bar{u} s$ structure, occurs at higher temperatures and is probably slowed down by the small decrease of the strange quark mass, $M_{s}$.

Concerning the axial symmetry, its effective restoration should be signaled by the vanishing of the observables related to the anomaly, like the gap between the masses of the chiral partners of the $\mathrm{U}_{A}(1)$ symmetry, and the topological susceptibility. For what concerns the observables here analyzed, we notice that although both models exhibit a tendency to a decrease of the anomaly effects, the restoration of the axial symmetry is not achieved: the masses of the partners $(\pi, \eta)$ and $\left(\sigma, a_{0}\right)$ although getting close at high temperatures, do not converge. We also verify that the mixing angles do not reach the ideal values [10]. This is expected since, in the framework of the NJL model, it has been shown that only with additional assumptions (for example, by choosing a temperature dependent anomaly coefficient [9] or using a regularization where the cutoff goes to infinity at $T \neq 0$ [12]) the restoration of the axial symmetry can be achieved.

We point out that the new feature of the PNJL model is that the faster decrease of the quark condensates leads to a faster partial restoration of chiral symmetry. In addition, although the axial chiral partners do not converge, in PNJL model the masses of $(\pi, \eta)$ become closer than in NJL, as well as those of $\left(\sigma, a_{0}\right)$. This is an indication that, although axial symmetry is not restored in the range of temperatures studied, the tendency to the possible restoration of this symmetry is faster in the PNJL model.

We also derive the topological susceptibility, $\chi$ (Fig. 2), that is far away from being zero in both models. However, it is interesting to notice that the PNJL calculation nicely reproduces the first lattice points, namely the rather steep drop around $T_{c}^{\chi}$, while this is not verified in the NJL model. Although restoration of axial symmetry is not achieved, this behavior of the topological susceptibility indicates that the PNJL model shows a more pronounced tendency for the restoration of the axial symmetry.

\section{Acknowledgments}

Work supported by Grant SFRH/BPD/23252/2005 (P. Costa) and by F.C.T. under projects POCI/FP/63945/2005 and POCI/FP/81936/2007 (H. Hansen).

\section{References}

[1] S. Datta, F. Karsch, P. Petreczky and I. Wetzorke, Phys. Rev. D 69, 094507 (2004).

[2] W.M. Alberico, A. Beraudo, A. De Pace and A. Molinari, Phys. Rev. D 72, 114011 (2005).

[3] H. Hansen, W. M. Alberico, A. Beraudo, A. Molinari, M. Nardi, and C. Ratti, Phys. Rev. D 75, 065004 (2007).

[4] P. N. Meisinger and M. C. Ogilvie, Phys. Lett. B 379, 163 (1996).

[5] E. Megias, E. R. Arriola, and L.L. Salcedo, Phys. Rev. D 74, (2006) 065005. 


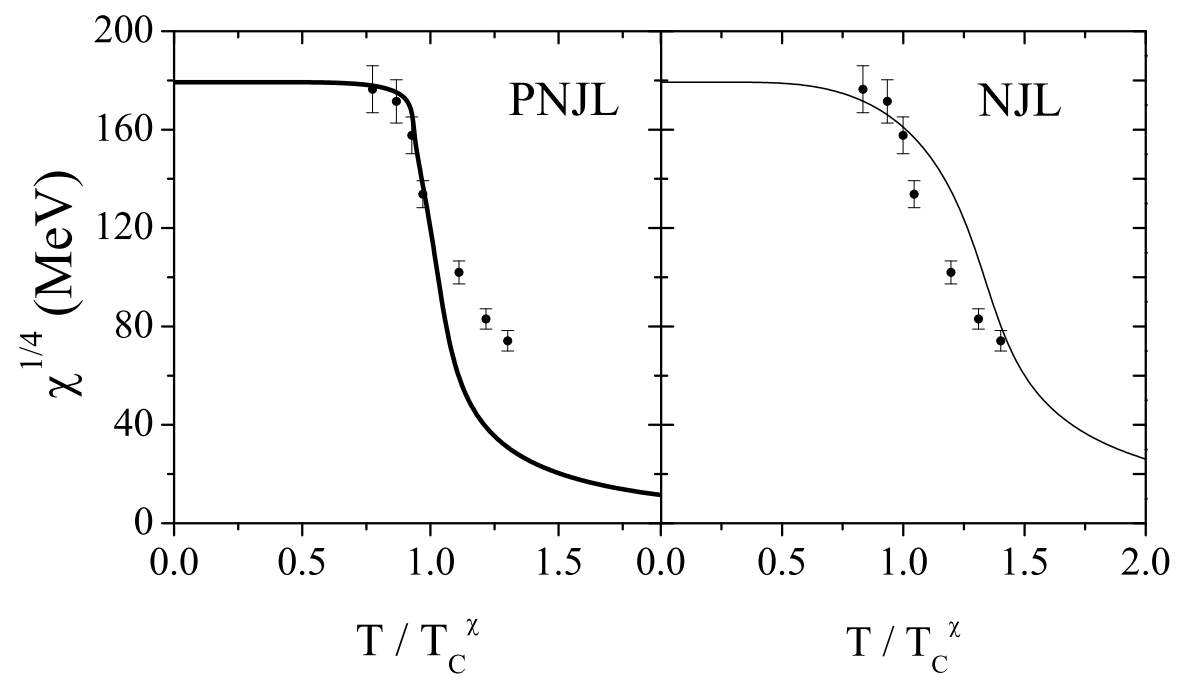

Figure 2: Topological susceptibility as a function of the reduced temperature $T / T_{c}^{\chi}$ for the PNJL (left panel) and the NJL (right panel) models.

[6] C. Ratti, M. A. Thaler, and W. Weise, Phys. Rev. D 73, 014019 (2006).

[7] Kenji Fukushima, Phys. Rev. D 77, 114028 (2008).

[8] A. M. Polyakov, Phys. Lett. B 72, 477 (1978).

[9] P. Costa, M. C. Ruivo, C. A. de Sousa, and Y. L. Kalinovsky, Phys. Rev. D 70, 116013 (2004); Phys. Rev. D 71, 116002 (2005).

[10] P. Costa, M. C. Ruivo, C. A de Sousa, H. Hansen, and W. M. Alberico, arXiv:0807.2134 [hep-ph].

[11] P. Costa, M. C. Ruivo, C. A de Sousa and Yu. L. Kalinovsky, Phys. Rev. C 70, 025204 (2004).

[12] P. Costa, M. C. Ruivo, and C. A de Sousa, Phys. Rev. D 77, 096009 (2008). 